\title{
Offsetting self-phase modulation in optical fibre by sinusoidally time-varying phase
}

\author{
Sonia Boscolo ${ }^{1}$, Frédéric Audo ${ }^{2}$, Misha Sumetsky ${ }^{1}$, and Christophe Finot ${ }^{2}$ \\ ${ }^{1}$ Aston Institute of Photonic Technologies, School of Engineering and Applied Science, Aston University, \\ Birmingham B4 7ET, United Kingdom \\ ${ }^{2}$ Laboratoire Interdisciplinaire Carnot de Bourgogne, UMR 6303 CNRS - Université de Bourgogne Franche- \\ Comté, F-21078 Dijon Cedex, France \\ e-mail: s.a.boscolo@aston.ac.uk
}

\begin{abstract}
We report on our recent experimental and theoretical results on the use of a sinusoidally time-varying phase to suppress undesirable self-phase modulation of optical pulses propagating in fibre-optic systems.
\end{abstract}

Keywords: Nonlinear fibre optics, phase modulation.

\section{INTRODUCTION}

The propagation of intense ultra-short optical pulses in a Kerr medium such as an optical fibre still remains a critical issue for the performance of many optical systems such as beam delivery, optical communication or pulse amplification systems. This is because the self-phase modulation (SPM) of the propagating pulse usually causes a broadening of the pulse spectrum that is typically accompanied by an oscillatory structure covering the entire frequency range [1]. Several strategies have been proposed and successfully deployed to counteract the deleterious effects of SPM in fibre-optic systems. These include spatial or temporal scaling to reduce the impact of nonlinearity via the use of very large mode area fibres [2], and the well-known technique of chirped pulse amplification [3]. A different class of approaches favours making the most of the Kerr nonlinearity. This class includes the exploitation of the peculiar properties of parabolic shaped pulses and self-similar evolution [4], the use of other types of pre-shaped input pulses, and the compensation of nonlinear phase shifts with third-order dispersion $[5,6]$. However, none of these last techniques preserves the pulse temporal duration.

A simple technique to compensate the nonlinear phase due to SPM and related spectrum broadening of nanosecond or picosecond optical pulses consists in using an electro-optic phase modulator to impart the opposite phase to the pulses. This method, which emulates the use of a material with a negative nonlinear index of refraction, has proved successful in fibre-optic and free-space optical telecommunication applications using phase-shift keying systems [7,8] and in the generation of high-peak-power nanosecond pulses [9]. In this paper, we report on our recent experimental and theoretical results in the area. We present a proof-of-principle experiment demonstrating that for input pulses with standard intensity profiles, such as Gaussian or hyperbolic secant pulses, the use of a simple sinusoidal drive signal for the phase modulator with appropriate amplitude and frequency is sufficient to reduce the nonlinear spectrum broadening to a large degree, and to significantly enhance the spectral quality of the pulses while their temporal duration remains unaffected [10]. Further, we present an in-depth characterisation of the SPM-mitigation method based on analytic results and numerical simulation of the governing equation. We assess the effects of the initial pulse shape and duration on the effectiveness of the technique, and we highlight the differences between pre- and post-propagation compensation schemes [11].

\section{PROOF-OF-PRINCIPLE EXPERIMENT}

For our proof of concept, we used the all-fibred experimental setup depicted in Fig. 1(a), which relies exclusively on commercially available components from the telecom industry. Our phase modulator is driven by a $\omega_{C}=25.1 \mathrm{GHz}$ rad electrical clock and imprints a sinusoidal phase $\varphi_{C}(t)=A_{C} \cos \left(\omega_{C} t+\varphi_{0}\right)$ with the appropriate phase offset $\varphi_{0}$ and amplitude $A_{C}$ on the optical wave. An effective cancellation of the nonlinear phase requires $\varphi_{0}=\pi$. The observed time dependence of the SPM-induced chirp of a Gaussian pulse propagating through a fibre in the nonlinearity-dominant regime suggests that this chirp can be offset in the central region of the pulse by choosing the angular frequency and amplitude of the modulation as: $\omega_{C}=\pi /\left(\sqrt{2} \cdot T_{0}\right)$ and $A_{C}=2 B \exp (-1 / 2) / \pi$. Here $T_{0}$ is the $1 / e$ intensity half-duration, $B=\gamma P_{0} z$ is the maximum nonlinear phase shift that occurs at the pulse centre and is widely known as the $B$-integral, $P_{0}$ is the peak pulse power, $\gamma$ is the coefficient of Kerr nonlinearity of the fibre, and $z$ is the propagated length $[10,12]$.

The experimental results obtained for $B=5 \mathrm{rad}$ are plotted in Fig. 1(b). The initial pulses are Gaussian-like in shape and have full-width at half-maximum (fwhm) duration of $120 \mathrm{ps}$. The temporal gradient of the pulse intensity numerically extracted from the experimental data shows that the SPM-induced chirp variation can be well approximated by a sinusoidal function, albeit featuring a small asymmetry. The pulse intensity profile at the fibre output is fully identical to the input, highlighting that with the fibre and temporal pulse durations being used, the propagation regime is purely nonlinear and is not affected by chromatic dispersion. Figures 1(c), (d) 
summarise the results obtained with and without phase pre-compensation for different pulse powers injected into the fibre up to $B=5 \mathrm{rad}$. While in the absence of phase correction we observe progressive expansion of the output spectrum from the fibre with increasing values of the accumulated $B$-integral, accompanied by the development of ripple and asymmetry, the use of a corrective phase modulation maintains an output spectrum that is only marginally affected. The benefits afforded by the phase correction method are also apparent when comparing the evolution of the root-mean-square (rms) spectral width of the pulse with the accumulated $B$ integral between the two configurations [Fig. 1(d)]. We have also assessed the effectiveness of the proposed approach in the context of Raman amplification, and observed that once again, the external phase modulation enables preservation of the spectral intensity profile to a large extent.
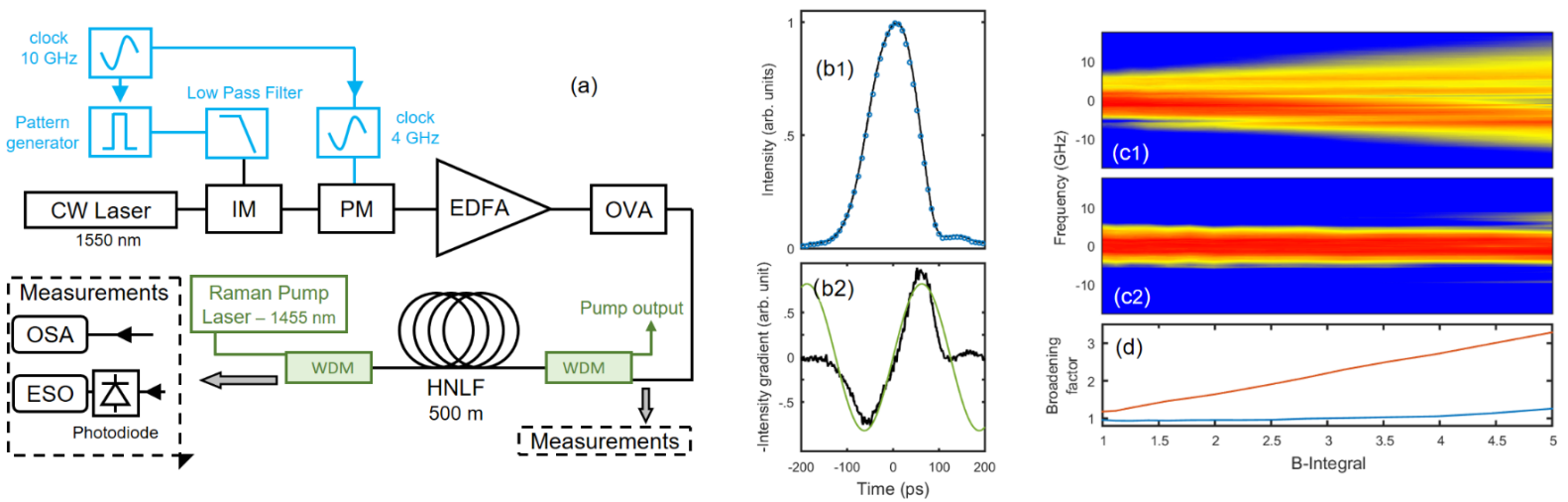

Figure 1. (a) Experimental setup. CW, continuous wave; IM, intensity modulator; PM, phase modulator; EDFA, erbium-doped fibre amplifier; OVA, optical variable attenuator; HNLF, highly nonlinear fibre; OSA, optical spectrum analyser; ESO, electrical sampling oscilloscope; WDM, wavelength multiplexer. (b) Experimental results for $B=5$ rad: (b1) Temporal intensity profiles of the pulse at the fibre input (black) and the input pulse with phase compensation (blue circles). (b2) Opposite of the temporal intensity gradient of the input pulse (black) compared with a sinusoidal fit (green). (c) Experimentally measured evolution of the frequency spectrum (logarithmic scale, 17-dB dynamic range) with the accumulated B-integral without (subplot 1) and with (subplot 2) phase compensation. (d) Evolution of the rms spectrum broadening factor with the B-integral without (red) and with (blue) phase correction.

\section{THEORETICAL ANALYSIS}

Propagation in the fibre was modelled by the standard nonlinear Schrödinger equation for the pulse envelope. We have numerically assessed the ability of our method to counteract SPM over a wide range of values of the $B$ integral accumulated in the fibre. The results obtained for an initially 100-ps-wide Gaussian pulse and the corrective phase modulation specified in the previous section are summarised in Figs. 2(a), (b), and highlight that the corrective approach can provide mitigation of SPM up to very high $B$ values: the fwhm of the pulse spectrum is largely preserved and no spectral ripple develops. The experimental results presented in the previous section are fully in line with these results. However, the cancellation of the nonlinear phase is not complete and the intensity level of spectral side lobes tends to grow with increasing accumulated $B$-integral. This is also reflected in the evolution of the rms spectral width of the pulse with the $B$-integral [Fig. 2(b)]: the rms spectrum broadening is reduced by a factor close to 4 relative to the case when no phase correction is used but it experiences a continuous increase with $B$ due to growing spectral side-lobes. The same trend is observed in the evolution of the spectral Strehl ratio, defined as the ratio of the maximum power spectral density (PSD) of the actual pulse to the PSD obtained assuming a transform-limited pulse and, thus, providing a measure of how close a pulse is to its Fourier transform limit. Following the calculation described in [13] and used by us in [14], we have derived an exact closed formula for the rms spectral width of an initially Gaussian pulse after undergoing SPM and with the corrective phase applied [11]. The rms spectral width evolution predicted by this equation is in perfect agreement with the numerical simulation results [Fig. 2(b)], and the equation explains the close to fourfold reduction in spectrum broadening at large $B$ as compared to the uncompensated case that is observed.

Our phase compensation method is capable of effectively counteracting the unwanted effects of SPM on the propagation of other pulse waveforms, provided that these can be fitted by a parabolic profile in their central part [14]. To corroborate this statement, we have illustrated the case of a hyperbolic secant pulse like those delivered by soliton lasers. Following the same idea of reducing the chirp in the central region of the pulse, one may derive the analogue of the formulae for $\omega_{C}$ and $A_{C}$ given in the previous section, for a hyperbolic secant intensity profile [11]. However, we have observed that while this choice of modulation parameters achieves fairly good chirp cancellation near the pulse centre, the deviations from perfect phase compensation on the pulse wings are rather 
strong, and these result in strong side lobes in the spectrum. On the other hand, for such pulse shape, optimisation of the parameters of the modulating sinusoid through a scan of the amplitude-frequency space outperforms the parameter choice based on the analytic guidelines, by triggering a threefold decrease in the rms spectrum broadening generated from SPM compared to the analytically calculated modulation and maintaining a high Strehl ratio at large $B$ values [11].
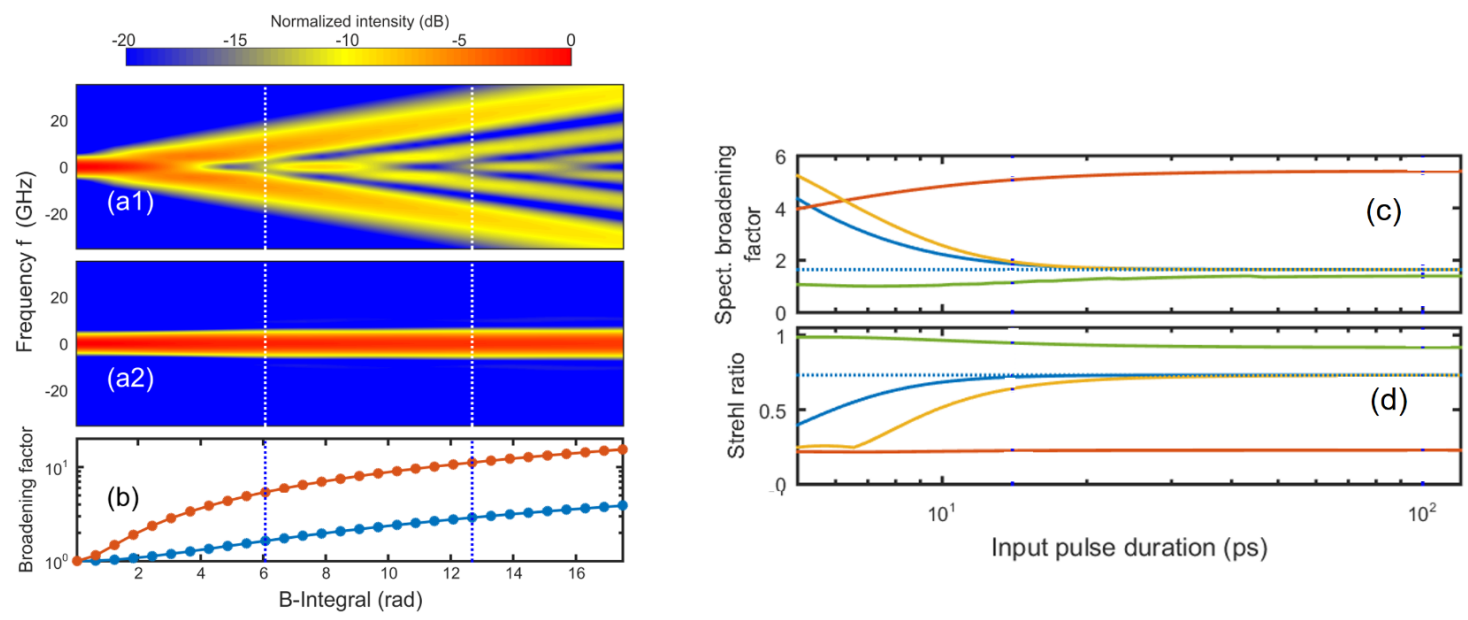

Figure 2. (a) Numerically computed evolution of the spectrum (logarithmic scale, 20-dB dynamic range) of an initial 100-ps Gaussian pulse with the accumulated B-integral, before (subplot 1) and after (subplot 2) phase compensation. (b) Evolution of the rms spectral broadening factor. The results obtained without and with phase correction are plotted with red and blue lines, respectively. The circles represent the predictions from the exact closed formulae for the rms spectral width. (c)-(d) Numerically computed evolutions of: (c) the rms spectral

broadening factor and (d) the Strehl ratio of an initial Gaussian pulse with the input pulse duration. The accumulated B-integral is $2 \pi$. The results obtained without phase modulation, with the phase modulation chosen

based on analytic design rules applied as a post-compensation, with the phase modulation chosen based on analytic design rules and applied as a pre-compensation, and with the optimised phase modulation applied as a post-compensation are plotted with red, blue, yellow and green lines, respectively.

Further, we have assessed the impact of the temporal duration of the initial pulses on the performance of our technique by varying the fwhm duration of a Gaussian input pulse from a few to a hundred picoseconds [Figs. 2(c), (d)]. First, we observe that initial temporal durations above $30 \mathrm{ps}$ do not affect the spectral metrics of the pulse, which pinpoints unaltered quality of the spectrum for these pulse durations. Similarly, the temporal intensity profile remains unchanged. This proves that the regime of propagation is dominated by Kerr nonlinearity with no influence of dispersion, and hence nonlinearity can be treated as being a lumped temporal phase modulator. The picture is rather different for shorter initial pulse durations, for which the interaction between nonlinearity and dispersion in the fibre is no longer negligible and so the pulse dynamics becomes more complex than the development of a simple temporal phase term. Two remarkable trends are captured in Figs. 2(c), (d): firstly, the analytic design rules for the parameters of the phase modulation given in the previous section become ineffective when dispersion comes into play. Secondly, while for a purely nonlinear propagation the disposition of the nonlinear element and the external phase modulator does not impact the overall SPMmitigation process, this is no longer the case when the pulse temporal intensity profile is affected by propagation in the fibre. The quality of the SPM-mitigation highly depends on the compensation scheme being used. A precompensation with parameters chosen according to the analytic formulae yields poor mitigation. Optimising the parameters of the sinusoidal modulation may help increase the spectral peak brightness and approach performances similar to those achieved with a post-compensation based on the parameters given by the analytic formulae. However, much better results both in terms of Strehl ratio enhancement and suppression of the residual side lobes in the spectrum can be obtained using a post-compensation with optimised parameters.

\section{CONLUSION}

We have presented a proof-of-principle experiment and an in-depth theoretical characterisation of the mitigation of the impact of SPM on optical pulses propagating through an optical fibre, relying on imparting a sinusoidal phase modulation to the pulses in the time domain. We believe that the present study can provide a deeper insight into the association of the SPM effects with an external sinusoidal phase modulation and can be of interest for multistage fibre amplifier architectures. We also expect that some features of the spectral compression process driven by SPM discussed in our previous work [15] can be readily extended to the SPM- 
mitigation scheme described in this paper so that the present scheme should be rather stable against amplitude fluctuations and signal-to-noise ratio degradation of the input pulses. We also believe that, similarly to a nonlinear spectral compression stage, the present technique could be combined with fibre laser architectures into new cavity designs $[16,17]$. Other prospected applications of the proposed approach include the reduction of different unwanted temporal phase defects such as those developed by pulses upon propagation in gain switched fibres or semiconductors devices.

\section{ACKNOWLEDGEMENTS}

We acknowledge financial support by the Région Bourgogne (Pari Photcom) and the Agence Nationale de la Recherche (Labex Action ANR-11-LABX-01-01). This work was also supported by the European Union within the framework of the operational Program FEDER-FSE Bourgogne 2014-2020 and by the Institut Universitaire de France.

\section{REFERENCES}

[1] R.H. Stolen, C. Lin: Self-phase modulation in silica optical fibers, Phys. Rev. A, vol. 17, pp. 1448-1453, 1978.

[2] X. Ma, et al: Sinlge-mode chirally-cupled-core fibers with larger than $50 \mu \mathrm{m}$ diameter cores, Opt. Express, vol. 22, pp. 9206-9219, 2014.

[3] D. Strickland, G. Mourou: Compression of amplified chirped optical pulses, Opt. Commun., vol. 56, pp. 219-221, 1985.

[4] C. Finot, C., et al.: Optical parabolic pulse generation and applications, IEEE J. Quantum Electron., vol. 45, pp. 1482-1489, 2009.

[5] L. Shah, et al.: High energy femtosecond Yb cubicon fiber amplifier, Opt. Express, vol. 13, pp. 4717-4722, 2005.

[6] S. Zhou, et al.: Compensation of nonlinear phase shifts with third-order dispersion in short-pulse fiber amplifiers, Opt. Express, vol. 13, pp. 4869-4877, 2005.

[7] C. Xu, L. Mollenauer, X. Liu: Compensation of nonlinear self-phase modulation with phase modulators, Electron. Lett., vol. 38, pp. 1578-1579, 2002.

[8] T. Ulmer, et al:: Mitigation of self-phase modulation in high-peak-power lasercom systems, in: Lasers Congress 2016 (ASSL, LSC, LAC), OSA Technical Digest (online) (Optical Society of America, 2016), LW3B.6.

[9] R. Su, et al.: High-peak-power, single-frequency, single-mode, linearly polarized, nanosecond all-fiber laser based on self-phase modulation compensation, Appl. Opt., vol. 52, pp. 7331-7335, 2013.

[10] F. Audo, et al.: Nonlinear spectrum broadening cancellation by sinusoidal phase modulation, Opt. Lett., vol. 42, pp. 2902-2905, 2017.

[11] S. Boscolo, F. Audo, C. Finot: Impact of initial pulse characteristics on the mitigation of self-phase modulation by sinusoidally time varying phase, Opt. Quant. Electron., 50:62, 2018. https://doi.org/10.1007/s11082-018-1319-1

[12] S. Boscolo, L.K. Mouradian, C. Finot: Enhanced nonlinear spectral compression in fibre by external sinusoidal phase modulation, J. Opt., vol. 18, 105504, 2016. doi:10.1088/2040-8978/18/10/105504

[13] S.C. Pinault, M.J. Potasek: Frequency broadening by self-phase modulation in optical fibers, J. Opt. Soc. Amer. B, vol. 2, pp. 1318-1319, 1985.

[14] S. Boscolo, et al.: Impact of initial pulse shape on the nonlinear spectral compression in optical fibre, Opt. Laser Technol., vol. 99, pp. 301-309, 2017.

[15] S. Boscolo, J. Fatome, C. Finot: Impact of amplitude jitter and signal-to-noise ratio on the nonlinear spectral compression in optical fibres, Opt. Commun., vol. 389, pp. 197-202, 2017.

[16] F.Ö. Ilday, F.W. Wise: Nonlinearity management: a route to high-energy soliton fiber lasers, J. Opt. Soc. Amer. B, vol. 19, pp. 470-476, 2002.

[17] S. Boscolo, S.K. Turitsyn, C. Finot: Amplifier similariton fiber laser with nonlinear spectral compression, Opt. Lett., vol. 37, pp. 4531-4533, 2012. 